\title{
Análisis del contexto e-learning en la UNAD. Una mirada desde los entes tecnológico, social y pedagógico
}

\author{
Clara Esperanza Bilbao ${ }^{1}$ \\ Lorena Marcela Velásquez ${ }^{2}$ \\ Diana Liceth Martínez ${ }^{3}$ \\ Alexander Fernández ${ }^{4}$
}

\begin{abstract}
Resumen
El presente análisis establece el estado actual del modelo e-learning a partir de los entes tecnológico, social y pedagógico en la Universidad Nacional Abierta y a Distancia (UNAD). El planteamiento del análisis se da a partir de los resultados de una exploración a las posturas conceptuales desde estudios empírico-analíticos, fenomenológicos y sistémicos a nivel global, que brindan los argumentos para dar una mirada a la institución desde la articulación tecnológica, social y pedagógica. El documento presenta la visión integral del e-learning a manera de introducción, establece los entes necesarios y posteriormente enlaza los mismos al contexto Unadista resaltando su articulación.
\end{abstract}

\footnotetext{
1 Licenciada en Idiomas de la Universidad Nacional (juniversidad nacional de dónde?), Especialista en traducción español - inglés - francés de la Universidad del Rosario y magister en Lingüística Aplicada a la Enseñanza del Español como Lengua Extranjera de la Universidad de Jaén (España). Tutora virtual de inglés de la UNAD desde el año 2007 y, a partir de 2011, tutora de la Licenciatura en Inglés como Lengua Extranjera. Es también tutora virtual de inglés certificada por el Consejo Británico y el Ministerio de Educación Nacional.

2 Profesional en Lenguas Modernas (Universidad EAN), especialista en Pedagogía para el Desarrollo del Aprendizaje autónomo (UNAD) y magister en Lingüística Aplicada a la Enseñanza de ELE (Universidad Jaén, España). Certificada como Tutor Virtual de inglés del Concejo Británico y MEN. Tutora y directora de curso del Programa Nacional de inglés, ECEDU, UNAD desde el 2007.

3 Licenciada en Español y Lenguas, Especialista en Docencia del Español y Magíster en Educación de la Universidad Pedagógica Nacional, actualmente cursa una especialización en Docencia Universitaria. Tutora virtual de inglés desde el año 2009 y, actualmente, tutora del programa de Licenciatura en Inglés como Lengua Extranjera, UNAD. Co-investigadora del grupo Virtualex de la Escuela de Ciencias de la Educación UNAD.
}

4 Licenciado en Administración Educativa de la Universidad de San Buenaventura en Cali (Colombia) y magister en Educación Superior de las Universidades Oslo (Noruega), Tampere (Finlandia) y Aveiro (Portugal). Tutor del Programa Nacional de Inglés ECEDU, UNAD desde el 2005. 
Los resultados reflejan posturas sobre el estado actual del contexto e-learning institucional.

Palabras clave: E-learning, entes, tecnológico, social, pedagógico.

\title{
Analysis of the e-Learning context in the UNAD an insight from the technological, social and pedagogical fields
}

\begin{abstract}
The present analysis establishes the current state of the e-learning model at the Open and Distance National University (UNAD) from the perspectives of the technological, social and pedagogical entities. The framing of the discussion is given from the exploration on conceptualizations after empirical analytical, phenomenological and systemic studies at the global level that allows for setting up the arguments to give a critical review to the technological, social and pedagogical articulation on the institution. The paper is organized by presenting an integral vision on e-learning, the establishing of the entities and connecting them to the Unadista context but highlighting their articulation. Results are the insights about the current state of the institutional e-learning context.
\end{abstract}

Key words: E-learning, entities, technological, social, pedagogical.

Recibido: 27 de enero 2012

Aceptado: 18 de mayo 2012

\section{lntroducción}

El término e-learning relaciona los procesos de enseñanza y aprendizaje mediados por el uso de las Tecnologías de la Información y la Comunicación (TIC). La educación electrónica ha sido interpretada desde diversas perspectivas permitiendo una amplia gama de concepciones y usos (Isamil, 2002; Mei-Yu, 2003; Kelly y Bauer, 2004; Rosenberg, 2006; Canadá, 2009) ${ }^{5}$. Según Fournier 
$(2006)^{6}$, el e-learning se ha convertido en una noción que se ajusta a diversas circunstancias para la aplicación de tecnologías computarizadas en la educación, ya sea en modelos híbridos, en contextos de educación a distancia o en contextos puros de e-learning.

Los modelos híbridos, también conocidos como blended learning, se refieren al uso alterno de modelos de educación tradicional y modelos de educación electrónica (online) dentro de un mismo contexto institucional con el objetivo de potencializar los encuentros presenciales en la educación tradicional. Por su parte, en la educación a distancia, el e-learning se combina brindando espacios alternos para encuentros tutoriales esporádicos inherentes a este modelo y en el uso intensivo de las TIC con propósitos formativos. Finalmente, el modelo de e-learning puro se concibe como la mediación total con los estudiantes a través del uso exclusivo de las TIC dentro del proceso de formación vocacional, profesional o de postgrado.

Aunque la implementación del e-learning ya completa cuatro décadas desde su inicio, su crecimiento exponencial reciente está directamente relacionado con el crecimiento del internet en la era actual de la globalización, la sociedad del conocimiento y la comunicación. En los años 60, la creación del computador revolucionó distintos campos del conocimiento y profundizó en la especialización de la investigación aplicada desde lo tecnológico. Alternamente, para los pedagogos y sicólogos, el potencial educativo del computador se convirtió en objeto de investigación desde la perspectiva de las ciencias puras.

Las dos perspectivas investigativas permitieron el desarrollo de sistemas de enseñanza inteligentes conocidos como Intelligent Teaching Systems (ITS) facilitando de manera funcional la generación de instrucción en tiempo real mediante los usos del computador. La investigación en ciencias aplicadas ayudó a fortalecer la automatización de los conceptos básicos de instrucción. Como resultado de la proliferación de los computadores personales, se incrementó el desarrollo masivo de los programas de instrucción multimedia con altos niveles de innovación tecnológica a través de la mediación por computador para el desarrollo de contenidos. Por su parte, la investigación desde las ciencias puras se enfocó en el desarrollo de modelos orientados en la estructura de la información con el objetivo de representar la cognición humana y el aprendizaje. Estudios previos como los de Datta y Ottmann (2001) desde las perspectivas de educación superior en Alemania y Australia, al igual que Nistor et. al. (2003) desde el contexto Rumano, permiten evidenciar la evolución alterna de la investigación, tanto en la educación a distancia como en la evolución del computador, y el internet mediante el recorrido histórico a los aportes hechos en ambas líneas.

5 Los autores y organizaciones citados definen e-learning desde diversas ubicaciones geográficas en el contexto de la educación superior.

6 Hélène Fournier expone una conclusión tras un análisis a las diferentes concepciones de e-learning desde la perspectiva Canadiense. 
Adicionalmente, los avances en el uso del internet provocaron el aceleramiento amplio de la investigación aplicada y pura en la línea de los modelos multimedia y la instrucción respectivamente, generando un fácil acceso a la información y al conocimiento. El ejemplo tácito de esta evolución con la aparición del internet se evidencia con la adaptación de los CD-ROM a sistemas de autoría basados en la red. Es decir, como resultado se unificó el contenido y el control de los mismos a través del surgimiento de sistemas de administración del aprendizaje basado en el computador. Hoy, en el siglo XXI, encontramos las plataformas educativas que permiten a las instituciones de educación en todos los niveles, hacer uso de ellas con intenciones formativas, de desarrollo de habilidades y de investigación, entre otras.

Tras la combinación de estrategias tecnológicas y educativas, las concepciones de e-learning se pueden interpretar desde las corrientes investigativas de las ciencias aplicadas y de las ciencias puras. Aunque para Fournier (2006), la concepción de e-learning está lejos de ser unificada, ello no necesariamente implica ambigüedad. E-learning reúne el potencial para proveer la información correcta a la población indicada en el tiempo y lugar preciso a través de un canal tecnológico (Rosenberg, 2006). Es decir, las estrategias tecnológicas y pedagógicas dentro del contexto e-learning se pueden evaluar para establecer cómo la instrucción es transmitida de manera electrónica a través de buscadores en red por medio del internet, intranet o plataformas multimedia (CD ROM, DVD, etc.) mediante el análisis de la articulación de los entes tecnológicos, sociales y pedagógicos que interactúan implícitamente en este contexto.

Es claro que el e-learning cubre una amplia gama de aplicaciones tales como el aprendizaje en red, aprendizaje mediado por el computador, aprendizaje a través del campus virtual, o simplemente por medio de ayudas digitales, incluyendo el uso de medios como internet, intranet, extranet y transmisión satelital interactiva, entre otros, para la transmisión de contenidos (Nistor et al. 2003). El análisis de la articulación de los entes arriba mencionados permite establecer el estado del contexto e-learning de una institución educativa, dado que lo tecnológico, lo social y lo pedagógico constituyen el ser del e-learning.

\section{Los entes dentro del contexto e-learning}

Se ha mencionado que los Sistemas de Enseñanza Inteligente facilitan de manera funcional la generación de instrucción en tiempo real mediante los usos del computador. En el momento actual, las instituciones de educación superior a nivel global, dentro del contexto e-learning, utilizan plataformas educativas con intenciones formativas. Dichas plataformas se comprenden como sistemas de administración de aprendizaje o como sistemas de administración de contenidos (Learning Management Systems LMS o Content 
Management Systems CMS). Estudios realizados por el Massachusetts Institute of Technology (MIT, 2006) evidencian que universidades de renombre mundial han desarrollado, a nivel institucional, sus propias plataformas para la administración de sus contenidos. También, algunas universidades, dentro de este grupo, han optado por el uso de plataformas comerciales o de libre acceso. El estudio también argumenta que el uso de plataformas refleja un incremento altamente significativo en la implementación de cursos (blended y online) en la última década. Los resultados muestran que 6 de las 11 instituciones, dentro de las mejores de los Estados Unidos que participaron en el estudio, utilizan plataformas comerciales.

A través de los LMS o CMS, las instituciones de educación superior proveen las plataformas para la generación de ambientes virtuales de aprendizaje (AVA), facilitando la administración, el suministro y el registro de las actividades de aprendizaje además de facilitar la interacción de sus académicos y estudiantes. Dentro de las plataformas comerciales o de libre acceso están Blackboard, Prometheus, B-Space, Classes 2 y Moodle, entre otras. Los contenidos permiten la organización institucional dentro de un ambiente virtual de tal manera que, tanto el personal administrativo como los académicos y estudiantes, tengan acceso a todo un complejo de recursos para las actividades pertinentes.

La contribución de las plataformas educativas ha permitido cambios radicales en el contexto de la educación en general, en la medida en que las actividades de enseñanza y aprendizaje ya no están restringidas al uso tradicional del salón de clase. El e-learning, a través del uso de medios para el aprendizaje (internet, audio, video, transmisión satelital, televisión, radio, CD ROM, entre otros), ha facilitado cruzar la barrera de la interacción física tradicional (Ozkan y Koseler, 2009). Ello ha fortalecido las estrategias para la masificación o universalización de la educación superior, dado que los estudiantes no necesariamente están obligados a compartir el mismo espacio físico. También, el avance en las estrategias de aprendizaje autónomo permite la interacción sincrónica y asincrónica de los actores de la comunidad educativa. Los argumentos anteriores pueden explicar la razón por la cual, las universidades han incrementado los niveles de inversión en recursos para el desarrollo e implementación de la tecnología con fines educativos.

La contribución de las plataformas educativas ha permitido cambios radicales en el contexto de la educación en general, en la medida en que las actividades de enseñanza y aprendizaje ya no están restringidas al uso tradicional del salón de clase. El e-learning, a través del uso de medios para el aprendizaje (internet, audio, video, transmisión satelital, televisión, radio, CD ROM, entre otros), ha facilitado cruzar la barrera de la interacción física tradicional (Ozkan y Koseler, 2009). Ello ha fortalecido las estrategias para la masificación o universalización de la educación superior, dado que los estudiantes no necesariamente están obligados a compartir el mismo espacio físico. También, 
el avance en las estrategias de aprendizaje autónomo permite la interacción sincrónica y asincrónica de los actores de la comunidad educativa. Los argumentos anteriores pueden explicar la razón por la cual, las universidades han incrementado los niveles de inversión en recursos para el desarrollo e implementación de la tecnología con fines educativos.

Ozkan y Koseler (2009) identificaron dos entes dentro del contexto e-learning. El primero es el ente social, desde la tesis de que el desarrollo tecnológico no reemplaza el desempeño humano. Esto se visualiza en el hecho de que los académicos y demás actores dentro de un contexto universitario, llevan a cabo la implementación formativa inherente a la educación superior, la única diferencia en este tipo de implementación, es que es realizada a través de canales tecnológicos. El segundo es el ente tecnológico, encargado de los asuntos relacionados con sistemas de calidad, internet e informática. Es decir, todo lo relacionado a software, hardware, estabilidad, seguridad, uso, diseño organizacional, administración de plataforma, y demás asuntos tecnológicos.

Desde otro contexto situacional, Govindasamy (2002) argumenta que siendo e-learning otra forma de enseñanza - aprendizaje, la trascendencia de este modelo no solo radica en formar profesionales en el tiempo y lugar adecuado. Más allá de dicho objetivo, el autor plantea que el valor real del e-learning yace en la habilidad docente de activar el atributo de formar profesionales con las competencias y conocimiento preciso. En otras palabras, e-learning debe ser un conjunto de recursos tecnológicos entendidos como AVA, mediado por actores con el perfil pertinente, para facilitar el desarrollo de las competencias y conocimientos idóneos dentro de una disciplina, mediante la inclusión de los principios pedagógicos que fortalezcan la formación de los estudiantes. En consecuencia, aquellos que dinamizan la formación de profesionales dentro del modelo e-learning, pueden ser adicionados a los dos entes propuestos por Ozkan y Koseler (2009) y obtener, así, una perspectiva de e-learning a partir de los entes tecnológico, social y pedagógico.

\section{Marco analítico}

A partir de lo establecido en la parte anterior, para analizar una institución de educación superior desde el contexto e-learning, es preciso interpretar la organización desde los entes tecnológicos, sociales y pedagógicos. Ello permite en esencia responder al interrogante: ¿cómo se han articulado los entes tecnológico, social y pedagógico dentro de un contexto e-learning para favorecer la formación de sus estudiantes?

El ente tecnológico responde a los usos de la plataforma y de los AVA utilizados en términos de software, hardware, sistemas de calidad y 
administración de los mismos. El propósito fundamental es facilitar que todos los canales tecnológicos presten un servicio eficaz para permitir la interacción entre estudiantes y profesores bajo una sistematización óptima de los contenidos.

Dentro del ente social, los actores fundamentales son los que interactúan en el proceso enseñanza-aprendizaje, es decir profesores, instructores, tutores, asesores académicos, como también quienes son el propósito fundamental de este engranaje: los estudiantes. La calidad de la instrucción sigue siendo un factor determinante dentro de la educación en sus diversas ecologías. Charpentier et al. (2006) enfatiza que los resultados del aprendizaje tienen efectos mayores si hay una buena implementación instruccional más allá de las ventajas tecnológicas.

Desde el ente pedagógico, es relevante considerar lo establecido por Govindasamy (2002) en otros contextos, dado que para el autor es claro en que los principios que aplican dentro del contexto de educación tradicional, también aplican dentro del modelo e-learning, pero deben ser extendidos a su funcionalidad dentro de los AVA. En últimas, las perspectivas pedagógicas facilitan el ajuste curricular de los programas, cursos, capítulos, lecciones y actividades propias del proceso enseñanza-aprendizaje.

Teniendo como base las interpretaciones anteriores, vemos que el contexto e-learning de la UNAD puede ser analizado desde los entes tecnológico, social y pedagógico, de forma que se haga visible el estado de la articulación de su modelo e-learning. El proceso de análisis, toma en primera parte, el contexto general de la UNAD con el propósito de conocer la trayectoria institucional desde sus inicios en la educación a distancia hasta la evolución del modelo e-learning actual. Como parte del contexto institucional, el análisis considera como referencia lo que se ha denominado en este documento el ente tecnológico, que en el contexto UNAD son: el campus virtual UNAD y los Objetos Virtuales de Aprendizaje (OVA), entre otros.

Por último, las perspectivas pedagógicas unadistas permiten comprender el rol del ente pedagógico en función de su articulación con los entes tecnológico y social. Los insumos del análisis son obtenidos desde la revisión de documentos institucionales, así como de la experiencia y observaciones realizadas por los investigadores que desarrollan este documento. Finalmente, las conclusiones discuten las condiciones actuales del modelo e-learning unadista.

\section{El contexto unadista}

La Universidad Nacional Abierta y a Distancia es una universidad pública colombiana fundada en 1981 (UNAD, 2007). Tras sus tres décadas, la UNAD puede ser considerada como la universidad estatal de mayor evolución 
en el contexto nacional, dado sus permanentes cambios en la búsqueda del mejoramiento educativo para los colombianos que más necesitan capacitación a nivel tecnológico, profesional y de posgrado, a través de cinco escuelas que comprenden programas en las disciplinas de las ciencias administrativas, humanas, educativas, agrarias e ingenierías.

La evolución institucional permanente ha vivido cambios desde los componentes pedagógico, organizacional, de carácter y de modalidad. Herrera (2011), tras el análisis del perfeccionamiento pedagógico institucional, permite identificar tres momentos de evolución institucional.

El primer momento está enmarcado hasta la primera mitad de los años 90, donde el modelo pedagógico institucional estaba centrado en la heteroestructuración del aprendizaje. Bajo este momento, la acción académica estaba centrada en la instrucción a través de módulos impresos y el aprendizaje era orientado con base en los contenidos. El segundo momento está comprendido entre el último lustro del siglo XX y el primer lustro del tercer milenio. El modelo pedagógico enfocaba la auto-estructuración del aprendizaje como estrategia fundamental centrada en los estudiantes. El currículo se centró en problemas desde las disciplinas, especialmente desde las ciencias humanas, educativas y administrativas. El tercer momento, que inició desde el 2004 hasta la fecha, evolucionó a la inter-estructuración del aprendizaje fomentando la interacción de académicos y estudiantes a través de grupos colaborativos mediante el uso intensivo de las TIC.

La evolución desde la perspectiva del marco organizacional está en coyuntura con el tercer momento de perfeccionamiento del modelo pedagógico. La consolidación del Proyecto Académico Pedagógico Solidario -PAPS(UNAD, 2008) permitió el ordenamiento institucional, especialmente en lo pedagógico y didáctico, e introdujo a la organización tanto la estrategia para el uso de los ambientes educativos virtuales, como las unidades académicas y administrativas que dinamizan el uso de las TIC con propósitos educativos. En síntesis, el PAPS institucionalizó y estructuró las entidades sociales y tecnológicas en el naciente contexto e-learning unadista.

En lo relacionado con la evolución dentro del carácter institucional, la UNAD adquirió la denominación de universidad ${ }^{7}$ en el 2007, después de más de dos décadas de ser «Institución Universitaria». Lo anterior tuvo implicaciones directas desde la concepción institucional de autonomía institucional, investigación, oferta de niveles de programas, cuerpo académico y proyección a mediano y largo plazo. 
En cuanto a la modalidad, la fase 3 consolida el modelo e-learning puro a la interacción pedagógica de la UNAD. Como se mencionó anteriormente, los inicios de la UNAD enfocaron un modelo de educación a distancia bajo la concepción del constructivismo social para la formación de tecnólogos, profesionales y especialistas. Tras la fase dos, el modelo pedagógico incorporó herramientas de las TIC a la interacción académica; los encuentros tutoriales en pequeños y grandes grupos se alternó con la interacción a través del Campus Virtual, fomentando los aprendizajes autónomo, colaborativo y significativo. En la fase 3, el modelo e-learning se consolida fomentando, además de los tres aprendizajes ya mencionados, la educación por competencias y por núcleos problémicos, en la línea de la teoría crítica.

\section{Ente tecnológico}

La entidad tecnológica a la luz de este análisis, se refiere a los recursos tecnológicos utilizados por la UNAD dentro de la consolidación del modelo e-learning. Los AVA en la institución están comprendidos dentro del Campus Virtual (CV) como también los Objetos Virtuales de Aprendizaje (OVA) y otras herramientas tecnológicas que facilitan la interacción académica entre los diferentes actores en el proceso de enseñanza-aprendizaje unadista.

El CV UNAD, también conocido como la «Plataforma», es el escenario básico para el desarrollo de los procesos de aprendizaje en la institución. La plataforma actúa como el LMS y CMS a través del sistema Moodle. Es decir, la UNAD utiliza una plataforma de libre acceso para el desarrollo de los procesos de aprendizaje dentro del cumplimiento de su misión formativa, investigativa y de proyección social.

Las acciones planificadas que se llevan a cabo en este escenario virtual con intención formativa radican en la oferta de cursos web, adicional a la implementación de OVA, simuladores y repositorios orientados a consolidar los procesos de aprendizaje estimulando la consolidación de redes pedagógicas. Al interior del CV UNAD se encuentran las diferentes aulas virtuales, equivalentes a los diferentes cursos ofertados por los programas de pregrado y posgrado, como base para implementar los procesos de acompañamiento a los estudiantes.

A su vez, la plataforma guarda los archivos de ofertas anteriores y permite espacios virtuales para la interacción docente que facilita las redes académicas para la planeación, mejoramiento e investigación orientados al diseño, desarrollo y ajustes de los cursos con lineamientos internacionales.

A través de la plataforma Moodle, la UNAD ha institucionalizado la estandarización de la estructura de la presentación de las aulas virtuales, 
permitiendo que todos los actores interactúen dentro de un AVA con normatividad y condiciones de calidad. Todos los cursos virtuales de la UNAD presentan opciones de herramientas que facilitan la administración, información, acceso a contenidos, desarrollo de actividades de aprendizaje, evaluación e interacción asincrónica entre los diferentes actores. Actualmente, la estructura del CV UNAD está siendo rediseñada para ajustarse a los nuevos planes de perfeccionamiento pedagógico en la línea de la educación por competencias y los núcleos problémicos que obligan a considerar la interacción de los diferentes actores de manera más sincrónica.

Con relación a las mediaciones comunicacionales, las mismas tienen como propósito general desarrollar, producir, realizar y divulgar, interna y externamente, contenidos de comunicación organizacional basados en tecnologías digitales y telemáticas para contribuir a la consolidación y fortalecimiento de los diferentes componentes de la institución reflejados en el PAP Solidario.

Los objetivos del CV UNAD consideran un ciclo estratégico que permite la planificación interpretada como cultura organizacional innovadora, la implementación de las actividades de divulgación académica, cultural e investigativa mediante canales tecnológicos (radio, televisión, webconferences, etc.), la aplicación de estándares de calidad en cada aplicación de procesos tecnológicos y la evaluación constante y sistemática de cada uno de sus procesos.

En relación con las innovaciones tecnopedagógicas, las mismas tienen como propósito general la producción de mediaciones pedagógicas, didácticas y de objetos virtuales de aprendizaje basados en tecnologías digitales y telemáticas, contribuyendo de manera específica al cumplimiento de la misión institucional y de sus responsabilidades formativas, investigativas y de proyección social. Es claro para la institución que dichas innovaciones tienen objetivos específicos que inician desde la conformación de redes académicas con intenciones formativas bajo lineamientos de calidad, hasta el de desarrollo de objetos virtuales de aprendizaje con intenciones formativas que facilitan que los estudiantes consoliden el aprendizaje.

La parte de innovación pedagógica es considerada dentro del CV UNAD de manera específica: las actividades académicas están enmarcadas dentro de una estandarización institucional que permite evaluar los resultados de aprendizaje de una manera secuencial a través de las fases de aprendizaje de reconocimiento, profundización y transferencia.

Con la visión secuencial de las fases de aprendizaje, la UNAD concibe el aprendizaje como un proceso de carácter cognitivo, psico-afectivo y motor que no termina, se realiza de forma permanente y permite el desarrollo 
de competencias para los seres humanos (UNAD, 2005). Desde esa perspectiva, para los procesos formativos en contextos educativos, las fases del aprendizaje representan una estrategia que asegura el aprendizaje y el desarrollo efectivo de la propuesta curricular inherente a los diversos programas en las diferentes líneas disciplinares.

Las fases del aprendizaje en la UNAD pretenden secuenciar los niveles de dominio de conocimiento en cada uno de los cursos ofertados para cada programa. Así, la primera fase es la de reconocimiento que permite identificar los conocimientos previos de los estudiantes desde un proceso cuantitativo y cualitativo con intención diagnóstica que permite a los actores que orientan el proceso de aprendizaje, para el caso de la UNAD: docentes o tutores, determinar las estrategias metodológicas y didácticas que secuencian el proceso de aprendizaje.

La siguiente fase es la profundización de los conceptos relevantes a cada curso académico permitiendo que los estudiantes aprehendan de forma crítica, significativa, colaborativa y autónoma los elementos conceptuales y pragmáticos pertinentes a los diversos cursos en los diferentes programas ofertados por la institución. Esta fase lleva a los estudiantes a alcanzar niveles de conocimiento profundos y reales acorde con la intención del curso.

Para terminar, la fase de transferencia permite evidenciar en los estudiantes niveles de apropiación y aplicación de los conceptos y experiencias a través de acciones prácticas desde el énfasis socio-comunitario de la UNAD.

Las fases del aprendizaje en la UNAD están institucionalmente definidas en relación a los créditos académicos, reflejados en horas de estudio y prácticas puntuales por cada fase. El mayor énfasis se hace en las fases de transferencia y profundización para asegurar un mayor aprendizaje.

Con respecto al e-learning, las fases del aprendizaje en la UNAD representan un elemento transcendental al incluir el uso de la tecnología en programas de educación superior. Dichas fases marcan el proceso a seguir dentro de un grupo de cursos de orden socio-humanístico, investigativo, básico disciplinar, de conocimiento específico y electivo, dispuestos a través del Campus Virtual institucional, donde los estudiantes consolidan su aprendizaje a través de un proceso secuencial que busca el desarrollo del pensamiento crítico y de competencias acordes con la disciplina y el énfasis socio-comunitario.

En síntesis, el CV UNAD es un canal tecnológico que inicia con la interacción de los diferentes actores del proceso de aprendizaje, desde el proceso de planeación académica y tecnológica, y culmina con la evaluación de los resultados de aprendizaje de los estudiantes en la fase de transferencia. 
Es importante recalcar que durante todo el proceso de aprendizaje en el CV, los estudiantes encuentran en sus aulas, Objetos Virtuales de Aprendizaje (OVA) que contribuyen al proceso didáctico en calidad de pieza o canal de conocimiento.

Los OVA son recursos educativos reutilizables, empleados en el aprendizaje apoyado por las tecnologías de la información y la comunicación dentro del sistema e-learning de la UNAD, que son accesibles para los estudiantes de manera ilimitada.

Los objetos están disponibles en repositorios y organizados a través de descripciones en metadatos, para ser identificados fácilmente permitiendo ensamblar unidades, capítulos y lecciones en cursos tanto a nivel unadista como en otros contextos a nivel nacional, regional y global.

De acuerdo con lo expresado por Garrison y Anderson (2003), es tácito que el resultado deseado de la educación es la construcción de estructuras coherentes de conocimiento que fortalezcan el aprendizaje, contrario a la asimilación de fragmentos de información poco útiles. Dentro de la articulación de los sistemas de aprendizaje y conocimiento en la UNAD, los OVA permiten la dosificación progresiva de lo que Garrison y Archer (2000) denominan la «transacción educativa». En otras palabras, es el ordenamiento que responde al cómo la información se transforma en conocimiento para el individuo a través de la interacción social mediada por las TIC.

La UNAD como institución educativa que desarrolla procesos de aprendizaje no presencial, asistidos por computador, cuenta con la Red OVA que se encuentra a cargo de la Vicerrectoría de Medios y Mediaciones Pedagógicas. Esta Red fue diseñada para que el personal académico de la universidad (docentes, tutores, consejeros, diseñadores, investigadores y estudiantes) disponga de una plataforma habilitada para la construcción y utilización de los OVA que fortalezcan los procesos formativos.

Así, los procesos de mejoramiento y control de calidad implementados por la UNAD contemplan una serie de reglas que se deben cumplir para alcanzar las metas de formación por medio del uso e implementación de OVA. Este proceso de mejoramiento va estrechamente ligado al programa de Formación de Formadores de la institución, destinado a formar personal que propenda por el cumplimiento de los objetivos académicos de los estudiantes de la UNAD. Dentro de los marcos existentes, la estructura de los cursos académicos de la UNAD está directamente ligada al número de créditos que representa dicho curso, donde cada crédito académico corresponde a 15 lecciones. Destacando lo anteriormente dicho, a una lección no corresponde un OVA, sino que corresponden todos los objetos que sean necesarios para garantizar la aprehensión del conocimiento. 
Lo importante es que el objeto cumpla con un propósito social, informativo, educativo y ante todo se plantee de manera atractiva y dinámica, con mayor flexibilidad de la que permite un HTML y con la posibilidad de navegarse y generar retroalimentación de la evolución de los estudiantes. Actualmente, la UNAD cuenta con 57 redes académicas de OVA.

\section{Ente social}

El ente social es el componente humano y social que cumple con la función de llevar a cabo todos los procesos desarrollados en la universidad, entre los cuales se encuentran lo pedagógico, lo tecnológico y lo social. Es así que esta entidad social está compuesta por «Mediadores» (conocidos como el cuerpo docente y estudiantil de la universidad) que hacen parte de los actores del Modelo Pedagógico e-learning de la UNAD. Entre ellos están los docentes, tutores, consejeros, formadores y el eje fundamental por el cual existe la universidad: los estudiantes. Debido a las recientes modificaciones de diseños, mediaciones, funciones, perfiles, etc., ha sido necesario redefinir el rol de cada uno de los actores anteriormente mencionados, generando nuevos escenarios e instancias de aprendizaje como se muestra a continuación:

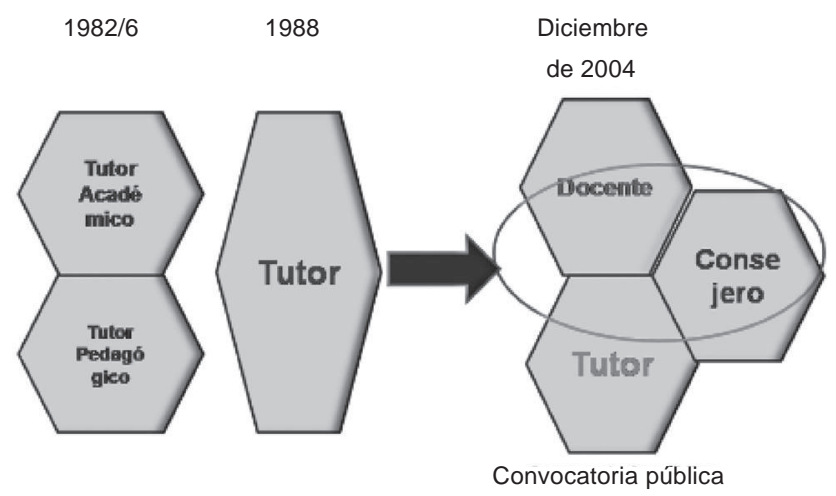

Figura 1. Nuevos roles del cuerpo académico. VIMMEP Fuente: Proyecto Académico Pedagógico Solidario (PAPS)

En primer lugar, se encuentran los consejeros que se destacan por desarrollar diferentes prácticas docentes y tutoriales en la institución. Por ejemplo, se concentran en el Sistema de Integración Unadista (SIU), entendido éste como un curso académico donde el estudiante que ingresa por primera vez a cualquier programa de pregrado, adquiere las concepciones, metodologías y técnicas para el estudio independiente y el aprendizaje autónomo como 
condición para asumir su formación a distancia. En este sentido, el rol del consejero tiene una importancia trascendental en las políticas que favorecen el mejoramiento de la calidad del aprendizaje en la formación a distancia y la retención de altos porcentajes de estudiantes en los programas. Algunas competencias laborales de los consejeros son: el seguimiento sistemático de los estudiantes para consolidar los procesos de estudio independiente, el diseño de pruebas con fines de evaluación de los procesos de aprendizaje de los estudiantes, el desarrollo de proyectos de investigación y el mejoramiento de las consejerías, como factor de calidad del aprendizaje y permanencia de estudiantes, entre otros.

En segundo lugar, están los tutores que están a cargo de realizar el acompañamiento tutorial en los procesos de aprendizaje y en la formación a distancia. Estos tienen un carácter pedagógico ya que son actores fundamentales de la Propuesta Pedagógica del Sistema del Área Académica de la UNAD. Los tutores realizan el acompañamiento mediante el seguimiento, evaluación y realimentación de las competencias que el estudiante va asumiendo, acorde con los parámetros establecidos por la institución. Asimismo, interactúan de manera dinámica con los directores y tutores de cursos afines mediante la creación de redes con el fin de consolidar estrategias de acompañamiento a los estudiantes y de mejoramiento de los procesos de aprendizaje.

Por otro lado, participan de manera activa en la elaboración y actualización de materiales didácticos de los cursos académicos a cargo, contribuyen en la elaboración de pruebas y apoyan los procesos de validación de las mismas, elaboradas por colegas. Además, diseñan y ponen en funcionamiento estrategias pedagógicas para contribuir a la creación de ambientes y escenarios favorables para el mejoramiento de la calidad del aprendizaje y participan en proyectos de investigación relacionados con la educación a distancia y el saber disciplinario en el que se desenvuelven, entre otros.

En tercer lugar, se encuentran los docentes, quienes según el Reglamento Académico, Art. 7., se definen como los actores educativos que centran su responsabilidad en el ámbito académico de la docencia, la investigación y la proyección social; desarrollan la planeación, diseño, seguimiento y actualización de currículo; elaboran y actualizan materiales didácticos y objetos virtuales de aprendizaje; realizan el diseño de estrategias e indicadores de acompañamiento; asesoran y hacen seguimiento a estudiantes; diseñan y elaboran sistemas e instrumentos de evaluación de procesos de aprendizaje y bancos de pruebas y además realizan el diseño de estrategias para las interactividades (UNAD, 2006).

En cuarto lugar, está el formador, otro rol significativo dentro de la universidad ya que tiene la responsabilidad de diseñar materiales que lleven a la cualificación y actualización del cuerpo académico de la UNAD, en las herramientas y 
temáticas necesarias para un excelente desempeño como actores académicos de la Institución. Además, los formadores crean, diseñan, elaboran y actualizan materiales didácticos de los cursos académicos en cada diplomado a ofertar; implementan, aplican y validan nuevas estrategias de aprendizaje en entornos virtuales. Los formadores dentro del programa de entrenamiento y cualificación docente ayudan a fortalecer la calidad académica y universitaria por medio del mejoramiento de los dispositivos pedagógicos de la formación a distancia donde se beneficiarán no sólo los estudiantes que realizan sus procesos de aprendizaje a través de la mediación del Campus Virtual, sino también aquellos que seleccionan la mediación por Sistema Tradicional, pues las diplomaturas y las certificaciones tienen como campo de aplicación las dos mediaciones.

Finalmente, se encuentra el estudiante, quien es el actor educativo más trascendental de la universidad y quien hace del aprendizaje autónomo la condición esencial para el desarrollo de la actividad académica, orientada a su formación Integral (UNAD, 2006). Asimismo, el PAPS resalta al grupo de estudiantes unadistas como una población totalmente heterogénea y multicultural, convirtiéndose en un valor agregado para la institución. Entre las competencias a desarrollar en el perfil estudiantil se encuentran: realizar procesos de reconocimiento del material didáctico de los cursos académicos, diseñar planes de trabajo académico para generar procesos de aprendizaje de profundización y apropiación de los contenidos didácticos; establecer líneas de trabajo para el desarrollo de actividades académicas, de transferencia, de conocimiento, de situaciones conocidas a situaciones desconocidas; definir procedimientos para la caracterización de sus ritmos de aprendizaje, estilos cognitivos y metodología de estudio con el propósito de mejorar y cualificar el tiempo de estudio, entre otros.

\section{Ente pedagógico}

La UNAD cuyo origen inicial se consideró bajo la denominación de «Unisur», nació ante la imperante necesidad de formación profesional para comunidades que por diversos motivos no podían acceder a la educación superior de tipo presencial, convirtiéndose así en la universidad piloto de la educación a distancia en Colombia. En consecuencia, la UNAD se ha visto en la necesidad de experimentar diversas transformaciones que le han permitido adaptarse a las necesidades de las poblaciones, proyectándose hacia unas perspectivas, que además de su compromiso social y académico, incorporan sistemas de calidad e innovación para las comunidades tanto locales como globales.

Bajo este panorama y respecto a los ámbitos pedagógico, didáctico y curricular, se ha hecho mención de los tres momentos de vital relevancia en el proceso de transformación unadista. El primero de ellos se centraba en el maestro y, 
por ende, privilegiaba la relación saber-docente así como la correspondiente transmisión de este saber mediante módulos impresos cuyos objetivos de aprendizaje se enfocaban en los contenidos. Así pues, esta etapa se centraba en la heteroestructuración del aprendizaje.

Empero, las subsiguientes tendencias educativas plantearon la necesidad de que los educandos dejaran de ser dependientes del profesor y de la situación instruccional ya que esto les proveía pocas herramientas a nivel cognitivo que les sirvieran para enfrentar, por sí mismos, nuevas situaciones de aprendizaje pertenecientes a distintos dominios y útiles ante las más diversas situaciones.

Es así como surge la idea de enseñar a los educandos a ser aprendices autónomos, independientes y autorregulados, capaces de aprender a aprender y, en consecuencia, surge un segundo momento en que el modelo pedagógico pasó de ser heteroestructurante para convertirse en un modelo autoestructurante donde el rol del estudiante es activo, participativo y constructor de conocimiento.

Desde esta perspectiva, el protagonismo del quehacer pedagógico se desplaza de la enseñanza y el docente al aprendizaje y al estudiante. En este sentido, se promovieron ambientes y situaciones que favorecían la participación del estudiante mediante una comunicación horizontal, privilegiando así la relación estudiante-saber, lo cual implicaba para el educando la capacidad de reflexionar en la forma en que aprende y actuar en consecuencia, autorregulando el propio proceso de aprendizaje mediante el uso de estrategias flexibles y apropiadas que se transferían y se adaptaban a nuevas situaciones.

Respecto al currículo, éste se enfocaba en problemas desde el punto de vista disciplinar (especialmente a partir de las ciencias humanas, educativas y administrativas) como punto de partida para la adquisición e integración de los nuevos conocimientos. De acuerdo con Exley y Dennis (2007), este enfoque curricular involucra un aprendizaje activo, centrado en el estudiante, asociado con un aprendizaje independiente muy motivado.

No obstante, en el trascurso de los años se percibió la necesidad de replantear el rol del maestro a fin de que éste hiciera parte más activa del proceso de enseñanza-aprendizaje como orientador. Es así como, a partir del año 2004, se acude a una estrategia metodológica de tipo interestructurante, la cual combina las cualidades positivas del modelo heteroestructural -como la mediación del maestro- y las cualidades del modelo autoestructural, ya que el rol de los estudiantes continúa siendo primordial en su propio proceso educativo a partir de sus iniciativas, medios, preguntas, interacciones, etc.

La adopción de esta perspectiva implicó, entre otras cosas, la interacción de académicos y estudiantes mediante el uso intensivo de las TIC. Se diseñaron y ofertaron cursos académicos virtuales montados en Moodle 
(entorno de aprendizaje dinámico modular) basados en las premisas del aprendizaje autónomo y la colaboración entre pares. Tales cursos presentan, fundamentalmente, dos divisiones en su estructura interna: la primera referida a los recursos de información y la segunda relacionada con las actividades a realizar, tanto por parte del estudiante como a nivel grupal.

Respecto a las actividades grupales, vale la pena aclarar que en cada curso virtual se consolidan pequeños grupos colaborativos -donde se asignan responsabilidades específicas-, a fin de que los estudiantes logren los objetivos planteados por el director de curso en un tiempo determinado -proceso que es orientado y constantemente monitoreado por un tutor-. Es importante destacar que esta interacción favorece el intercambio de los conocimientos que cada uno de los participantes tiene para aportar.

Esta estrategia metodológica interestructurante, consolidada desde el modelo pedagógico unadista, se cimentó en el enfoque epistemológico críticosocial e interpretativo, en directa relación con las teorías constructivistas e interaccionistas que tienen en cuenta la influencia del contexto social en que se aprende, así como los conocimientos previos y la historia del educando. A este respecto, es posible hacer alusión a teorías como: «aprendizaje significativo» de Ausubel (1983) y «mediación semiótica y zona de desarrollo próximo» de Vygotsky (1985). En consonancia con lo anterior, en este tercer momento se reestructuró el currículo de manera tal que se adoptaron normas internacionales, entre las que se cuenta el sistema de créditos académicos, así como la incorporación del componente de formación investigativa.

Actualmente, como proyección a la consolidación de un modelo e-learning, la universidad está trabajando en la reconstrucción de su enfoque pedagógico, en busca de la articulación de procesos de enseñanza- aprendizaje en torno a núcleos problémicos, a fin de fomentar la reflexión crítica y sistemática en la exploración de alternativas de solución a problemas específicos de cada disciplina. Todo ello, vinculado al desarrollo de competencias que integren el saber conocer, el saber hacer y el saber ser, con miras al mejoramiento social, a través de redes que integren a todos los actores pertenecientes al ente social.

\section{Análisis y discusión}

El propósito de este análisis es dar una mirada a cómo se han articulado los entes tecnológico, social y pedagógico dentro del contexto e-learning de la UNAD. Varias posturas se han presentado a lo largo del documento donde se plantean fundamentos sobre la educación electrónica. Es claro que la UNAD se encuentra en un momento de transición desde un modelo híbrido, combinando los cursos con encuentros tutoriales programados con estrategias presenciales 
y los cursos a través del campus virtual hacia un modelo e-learning puro; es decir, cursos mediados totalmente a través de AVA.

Los fundamentos teóricos del documento ayudan a suponer que un contexto e-learning puede ser analizado a través de los entes tecnológico, social y pedagógico donde se resaltan las posturas tecnológicas y pedagógicas desde las líneas de investigación aplicada y propiamente dicha. Desde el contexto unadista, se puede evidenciar dicha articulación, dado que el uso del computador y del internet han sido implementados en la institución, con fines formativos a través de un sistema educativo que está en la búsqueda de su perfeccionamiento pedagógico.

De acuerdo con lo planteado por Herrera (2011), la institución ha vivido tres momentos en la búsqueda del perfeccionamiento pedagógico. Específicamente, el tercer momento marca los inicios y la consolidación del modelo e-learning en la institución. En consecuencia, la implementación del modelo e-learning ha ocasionado cambios al interior de todos los componentes que hacen parte del PAP Solidario unadista. Ello está en concordancia con de Freitas y Oliver (2006) quienes desde el contexto británico han argumentado una relación directa entre la implementación del e-learning a nivel institucional, el cambio organizacional y las políticas de e-learning. En el caso específico de la UNAD, la implementación de este ha obligado a generar políticas institucionales para su aplicación en diversos procesos, las cuáles han llevado al cambio institucional. Dentro de las prioridades, está llegar a definir un modelo pedagógico concordante con el contexto e-learning. Todo apunta a que, además de darle continuidad a la profundización en los axiomas del aprendizaje autónomo y el aprendizaje colaborativo, también el modelo pedagógico incluirá los enfoques de la educación por competencias y por núcleos problémicos en línea con las perspectivas de la teoría crítica.

Otros cambios institucionales se reflejan en la integración de nuevas unidades en los niveles de la alta y la meso gerencia. Tal es el caso de la nueva vicerrectoría de internacionalización, la creación de un instituto virtual de lenguas y la apertura de nuevos programas de pregrado pensados bajo la mediación exclusiva del e-learning, como también la construcción de edificios inteligentes con alta inversión en tecnología. Ello puede implicar que no todos los cambios sean en relación directa con el e-learning, pero la orientación de nuevos planes y estrategias han sido favorecidos con la implementación del mismo. Como ejemplo, la proyección institucional hacia una mega universidad es dada a partir de las facilidades de espacio y tiempo que permite el e-learning. Desde ese sentido, el e-learning facilita la masificación de la educación superior $\mathrm{y}$, por ende, la proyección institucional desde diversas estrategias.

Como fundamento de este análisis, se han presentado posturas como las de Fournier (2006), quien argumenta sobre las diferentes conceptualizaciones 
sobre e-learning y aunque, desde la visión de los autores que construyen este documento, ello no genera ambigüedad; es preciso que el e-learning lleve la información indicada en el tiempo y lugar preciso a través de un canal tecnológico, tal como lo argumenta Rosenberg (2006). Sin embargo, dentro de la dinámica de llevar información en el tiempo y lugar preciso, el rol del ente social es fundamental dado que es el encargado de articular lo tecnológico y lo pedagógico. Por lo tanto, el dominio de la articulación de los tres entes en el contexto e-learning debe darse desde el ente pedagógico, mediado por el ente social a través del ente tecnológico como canal de comunicación.

No obstante, en el caso de la UNAD, el dominio se percibe desde el ente tecnológico. Ello se puede explicar desde el fenómeno que los entes sociales y pedagógicos, precisamente en ese orden, parecen haberse ajustado a las posibilidades que brinda el ente tecnológico representado en el CV UNAD a través de la plataforma Moodle. Como evidencia de lo anterior, se encuentra que la tercera fase de perfeccionamiento pedagógico inició con la vinculación del campus virtual a la actividad formativa institucional. Una vez se implementó el $\mathrm{CV}$, los mediadores del ente social dieron inicio a la estrategia de formación de formadores, con el objetivo de desarrollar las habilidades digitales pertinentes al manejo de plataformas educativas, para poder así facilitar y guiar a los estudiantes en su proceso de aprendizaje. En la fase actual de consolidación del e-learning, la institución trabaja en la adopción de un modelo pedagógico orientado a la educación por competencias y por núcleos problémicos en línea con la teoría crítica. Factores como la adopción reciente de este modelo y la proliferación del contexto e-learning en el mundo, arriba argumentado, justifican la implementación del mismo en la UNAD con propósitos formativos. En resumen, la UNAD está en la búsqueda del perfeccionamiento pedagógico dentro del contexto e-learning, lo que puede generar el dominio del ente pedagógico sobre el ente tecnológico una vez se alcancen mayores niveles de mejoramiento. Desde la anterior conclusión, se visualiza la continuidad de cambios a futuro para la consolidación del e-learning, como modelo formativo en la UNAD en su proceso de perfeccionamiento pedagógico.

En la articulación de los entes tecnológico, social y pedagógico desde el nuevo modelo formativo en la UNAD, se evidencia un funcionamiento sistematizado desde el ente tecnológico. Los sistemas de administración de contenidos y de aprendizaje están estandarizados y secuenciados acorde con la estructura organizacional unadista. Es claro que el CV UNAD proporciona los canales tecnológicos para permitir la interacción del ente social representado por los consejeros, tutores, docentes y formadores, bajo la estandarización de los contenidos y las actividades de aprendizaje. Sin embargo, estas actividades están sistematizadas de igual forma para todas las escuelas de formación en la institución. Ello se convierte en un paradigma que puede proponerse como tema de investigacion relevante que tendría como objeto evaluar el impacto que pueda reflejar un sistema de aprendizaje estandarizado para todas las 
disciplinas del conocimiento, que hacen parte de las áreas de formación en la universidad, y considerando la clasificación de las disciplinas establecidas por autores como Biglan (1973), Kolb, (1988), Becher (1989) quienes coinciden que las diferentes disciplinas del conocimiento se diferencian en sus sistemas de aprendizaje.

Por su parte, el ente social ha experimentado un crecimiento en su idoneidad para articular los entes tecnológico y pedagógico dentro de un modelo formativo a nivel universitario, bien en calidad de consejero, tutor, docente o formador, como también en calidad de estudiante. En consecuencia, se aprecia la gran contribución de la UNAD dentro del contexto colombiano, al potencializar las habilidades digitales de los actores de su comunidad universitaria. Es tácito que los integrantes del ente social de la UNAD deben obligatoriamente adquirir habilidades digitales desde el primer momento que se convierten en parte de la institución bajo cualquier rol, lo que acelera el proceso de alfabetización digital en el país. Es de presumir que las habilidades digitales adquiridas por los integrantes del ente social de la UNAD tengan un efecto colateral más allá de la institución. Estudios fenomenológicos pudiesen ayudar a descifrar el impacto institucional en el contexto colombiano con relación al incremento de habilidades digitales. Un alto impacto en el desarrollo de las habilidades digitales a nivel social en un país, facilitará la implementación del e-learning como modelo de formación y en consecuencia la disminución de la brecha de acceso a la educación superior en países como Colombia.

El ente pedagógico en el contexto e-learning de la UNAD, ratifica lo establecido por Govindasamy (2002), dado que a pesar del momento de búsqueda de perfeccionamiento y consolidación del modelo pedagógico en la UNAD, los postulados nacen desde corrientes tradicionales en lo referente a modelos pedagógicos, lo que se busca es que dichos modelos sean compatibles en contextos mediados por AVA. El modelo pedagógico de la UNAD, a pesar de su fase transitoria y en evolución, permite la interacción en AVA de su estructura curricular. Es claro que la institución está en búsqueda de su perfeccionamiento curricular, lo que puede facilitar que el ente pedagógico sea el ente dominante, en la medida que se continúe en la consolidación de un modelo pedagógico institucional.

Desde lo anterior, se aprecia que la implementación del e-learning en la UNAD llevó a la aplicación de actividades en AVA, la creación de políticas que facilitaran su aplicación y el posterior cambio institucional. Si bien, el inicio del e-learning llevó a la consolidación del ente tecnológico y secuencialmente a la redefinición de los entes social y pedagógico; actualmente se vive un proceso inverso que puede explicarse desde la búsqueda del modelo pedagógico compatible con AVA. Una vez se consolide el ente pedagógico dentro de la UNAD, el mismo podría alcanzar el carácter de ente dominante en relación al ente tecnológico. Si ello ocurre, el cambio institucional será en ese momento 
desde lo pedagógico a lo tecnológico y conllevará a reconsideraciones de la pertinencia del campus virtual actual, el rol de los OVA, el impacto de las actividades sincrónicas y asincrónicas, herramientas de comunicación entre los actores del ente social, al igual que las fases de aprendizaje para las diversas escuelas de formación. Se asume desde este análisis que el perfeccionamiento pedagógico provocará una reconsideración de la estructura de los LMS y CMS y por ende, del modo de interactuar del ente social bajo un nuevo ente pedagógico dominante.

\section{Conclusiones}

El analizar la articulación de un contexto e-learning a través de los entes tecnológico, social y pedagógico permite visualizar el estado actual de una institución de educación, que para efectos del documento actual, corresponde al nivel superior. Al dar una mirada a la luz de los tres entes, se puede identificar el ente dominante. Se asume que el dominio de los entes puede estar desde lo tecnológico o desde lo pedagógico. El ente social, en ambos casos, es quien articula la interacción a través de un canal tecnológico con carácter formativo. En el caso que el dominio del ente esté a favor de lo tecnológico, los LMS o CMS serán ajustados en la medida que los AVA lo permitan y a él también será ajustado el ente pedagógico. Para la situación opuesta, en la condición que el ente pedagógico sea el ente dominante, los AVA serán articulados a las condiciones que el ente pedagógico demande y el ente social interactuará a través de AVA pero con más fundamentos desde una perspectiva pedagógica.

En general, al vislumbrar los tres entes se aclara que el e-learning no es un modelo pedagógico per se; por el contrario, puede alcanzar el carácter de modelo formativo, siempre que esté acompañado por una interacción clara del ente social y bajo condicionamientos claros del ente pedagógico, que para dicho efecto será el modelo pedagógico en una institución de educación superior inmersa en contextos e-learning.

El estudio de los entes tecnológico, social y pedagógico puede originar interrogantes de carácter investigativo al interior de contextos e-learning que permitan fortalecer el carácter formativo de una institución de educación superior. Problemas tales como: ¿Cuál es el ente dominante en un contexto e-learning?, ¿Cuál enfoque pedagógico puede interpretarse como compatible dentro de un contexto e-learning?, ¿Cómo optimizar el rol del ente social dentro un contexto e-learning?, ¿Cuál es el impacto del modelo formativo e-learning en el contexto social donde se ubica la institución?, ¿Cuánto cambio es necesario dentro del contexto e-learning de la institución? son presentados como sugerencia a partir de los insumos del análisis de reflexión aquí presentado. 


\section{Referencias Bibliográficas}

Alger, B. 2009. The Experience Designer: Learning, Networks and the Cybersphere. Arizona: Fenestra Books.

Ausubel, D.P., J. Novak y H. Hanesian. 1983. Psicología educativa: un punto de vista cognoscitivo. México: Trillas.

Battezzati, L. 2002. E-Learning for Teachers and Trainers: Innovative Practices, Skills and Competences. Luxemburgo: Office for Official Publications of the European Communities.

Bautista, G., F. Borges y A. Forés. 2006. Didáctica universitaria en entornos virtuales de enseñanza-aprendizaje. Madrid: Narcea S.A. Ediciones.

Becher, T. 1989. Academic Tribes and Territories. Bristol PA: Society for Research into Higher Education and Open University Press.

Biglan, A. 1973. "The Characteristics of Subject Matters in Different Academic Areas". Journal of Applied Psychology, 57:3:195-203.

Canada. 2009. Canadian Council on Learning. State of E-learning in Canada. Ottawa.

Capacho, J. 2011. Evaluación del aprendizaje en espacios virtuales- TIC. Bogotá: Editorial Universidad del Norte.

Charpentier, P., C. Lafrance y G. Paquette. 2006. International E-learning Strategies: Key Findings Relevant to the Canadian Context. Ottawa: Canadian Council on Learning. Accessible en: www.ccl-cca.ca.

Datta, A. y T. Ottmann. 2001. "Towards a Virtual University”. Journal of Universal Computer Science. Vol. 7:10:870-885. Accesible en: http://www.jucs.org/jucs_7_10/ towards_a_virtual_university/Datta_A.html de Freitas, S. y M. Oliver. 2006. “Using Games and Simulations for Supporting Learning”. Learning Media and Technology. Vol. 31:4:343-358.

Díaz, F. 2002. Didáctica y currículo: un enfoque constructivista. Madrid: Universidad de Castilla- La Mancha.

Exley, K. y R. Dennis. 2007. Enseñanza en pequeños grupos en educación superior. Madrid: Narcea.

Fournier, H. 2006. A Review of the State of the Field of Adult Learning: E-Learning. Ottawa: Canadian Council on Learning. Accesible en: www.ccl-cca.ca. 
Friedland, G. 2007. Interactive Technology and Smart Education. Emerald Group Publishing Limited, Volume 4, Number 4, 104-198. Accessible en: http://bibliotecavirtual. unad.edu.co:2055/lib/unad/docDetail.action?docID=10229738\&poo=elearning

Garrison, D. R. y T. Anderson. 2003. E-Learning in the 21st Century. A Framework for Research and Practice. New York: Routledge. Accesible en: http://dl.acm.org/citation. $\mathrm{cfm}$ ?id $=582490$

Garrison, D. R., y W. Archer. 2000. A Transactional Perspective on Teaching and Learning: A Framework for Adult and Higher Education. Oxford, UK: Pergamon.

Govindasamy, T. 2002. "Successful Implementation of E-learning. Pedagogical Considerations". The Internet and Higher Education. Vol. 4:3-4: 287-299. Accesible en: http://www.sciencedirect.com/science/article/pii/S1096751601000719

Hannan, A. y H. Silver. 2005. La innovación en la enseñanza superior. Enseñanza, aprendizaje y culturas institucionales. Madrid: Narcea S.A. Ediciones.

Herrera. G.C. 2011. Ambientes virtuales de aprendizaje. Vicerrectoría de Medios y Mediaciones Pedagógicas. Bogotá: UNAD.

Horton, W. 2010. Designing Web-Based Training: How to Teach Anyone Anything Anywhere Anytime. Nueva York: John Wiley \& Sons, Ltd.

Ibernón, F., M. Alonso, M. Arandia, I. Cases, G. Cordero, I. Fernández, P. Revenga y P. Ruiz. 2002. La investigación educativa como herramienta de formación del profesorado. Barcelona: Gráo.

Isamil, J. 2002. "The Design of an e-learning System Beyond the Hype". The Internet and Higher Education. Volumen 4:329-336.. Revisado en http://www.qou.edu/arabic/ researchProgram/eLearningResearchs/designElearningSystem.pdf

Johnson Lehmann, K. 2009. How to be a Great Online Teacher. Nueva York: Rowman \& Littlefield Pub Inc.

Kapp, K. 2008. Winning E-Learning Proposals: The Art of Development and Delivery. Boca Raton Fla.: J. Ross Publishing.

Kelly, T. y D. Bauer. 2004. "Managing Intellectual Capital - via e-learning at Cisco". En: Handbook on Knowledge Management 2 Knowledge Directions. International Handbooks on Information Systems. Pp. 511-532. C.W. Holsapple. University of Kentucky, USA.

Kolb, D.A. 1988. "Learning Styles and Disciplinary Differences". En: The Modern American College. A.W. Chickering and Associates. Pp. 232-255. San Francisco: Jossey-Bass. 


\section{Clara Bilbao, Lorena Velásquez, Diana Martínez y Alexander Fernández}

Análisis del contexto e-learning en la UNAD. Una mirada desde los entes tecnológico, social y pedagógico, artículo de revisión

Marcelo, C. 2004. E-learning-teleformación. Diseño, desarrollo y evaluación de la formación a través de Internet. Barcelona: Ediciones Gestión 2000, S.A.

McConnell, D. 2006. E-Learning Groups and Communities. New York: McGrawHill Education. Accessible en: http://bibliotecavirtual.unad.edu.co:2055/lib/unad/ docDetail.action?docID=10172358\&poo=elearning

McKay, E. 2008. Human-Dimensions of Human-Computer Interaction. Amsterdam: IOS Press. Accessible en: http://bibliotecavirtual.unad.edu.co:2055/lib/unad/docDetail.ac tion?doclD=10267478\&poo=elearning.

Meyer, K. 2008. Quality in Distance Education: Focus On Online Learning. San Francisco: Jossey-Bass.

Wang, M.-Y. 2003. "The strategic role of digital libraries: issues in e-learning environments”. Library Review, Vol. 52:3:111 - 116. Revisado en http://www. emeraldinsight.com/journals.htm?articleid=859721\&show=abstract.

MIT. 2006. Massachusetts Institute of Technology. WCET Study: Peer Comparison of Course/Learning Management Systems and Related Costs. Final Report. Accesible en: www.wcet.info

Morrison, D. 2007. E-Learning Strategies: How to Get Implementation and Delivery Right First Time. Nueva York: John Wiley \& Sons, Ltd.

Nistor, N., S. English, S. Wheeler, y M. Jalobeanu (Eds.). 2003. Toward the Virtual University. International Online Perspectives. Greenwich, CT, EE.UU: Information Age Publishing.

Not, L. 2002. Las pedagogías del conocimiento. México: Fondo de Cultura Económica.

Ozkan, S. y R. Koseler. 2009. “Multi-Dimensional Students' Evaluation of E- Learning Systems in the Higher Education Context: an Empirical Investigation". Computers and Education. 53: 1285-1296.

Pedraza, R. y B. García. 2005. Flexibilidad académica y curricular en las instituciones de educación superior. México D.F.: Miguel Ángel Porrúa.

Ramón, M. A. 2004). El material didáctico y el acompañamiento tutorial en el contexto de la formación a distancia y el sistema de créditos académicos. Bogotá: UNAD.

Ramón, M. A. y E. Vidal. 2007. Balance social UNAD 2004-2007. Bogotá: UNAD.

Rosenberg, M.J. 2006. Beyond E-Learning. Approaches and Technologies to Enhance Organizational Knowledge, Learning and Performance. San Francisco: Editorial Pfeiffer. 
Salazar, R. 2011. Componente pedagógico-didáctico. Bogotá: UNAD.

Salmon, G. 2005. E-tivities: The Key to Active Online Learning. Londres: Taylor \& Francis Group.

Torre, J. 2002. Aprender a Aprender y Pensar para Aprender. Madrid: Narcea S.A. Ediciones.

UNAD. 2008. Universidad Nacional Abierta y a Distancia. Proyecto Académico Pedagógico Solidario (PAPS). Accesible en:http://demo.unadvirtual.org/moodle/ $\mathrm{mod} /$ resource/view.php?id=492

UNAD. 2007. Universidad Nacional Abierta y a Distancia. Información Institucional. Reseña Histórica. Accesible en: http://www.unad.edu.co/home/index.php/ resena-historica

UNAD. 2006. Reglamento Académico, Parte 08. Acuerdo Número 015 del 13 de Diciembre de 2006. Accesible en: http://www.unad.edu.co/secretaria/index. php?option=com_remository\&Itemid=\&func=startdown $\&$ id $=372$

UNAD. 2005. Universidad Nacional Abierta y a Distancia. Proyecto Académico Pedagógico de la UNAD. Accesible en: http://www.unad.edu.co/viacademica/ images/documentos/Viacademica/DocumentosViraci-2008/El\%20Proyecto\%20 Acad\%C3smico\%2oPedag\%C2\%BEgico\%20de\%2ola\%20\%20UNAD\%2odesafio\%20y\%20 exigencias\%2oregionales\%20en\%20su\%20aplicaci\%C2\%BEn.pdf

UNAD. 2004. Universidad Nacional Abierta y a Distancia. Inducción Unadista. Bogotá, D.C., Colombia.

Vygotsky, L. 1993. Pensamiento y lenguaje. Comentarios críticos de Jean Piaget. Buenos Aires: Ediciones Fausto. 